\title{
INFLUENCIA DE LA REGENERACIÓN DE PLANTACIONES SOBRE LA DIVERSIDAD Y DENSIDAD DE MACROINVERTEBRADOS EDÁFICOS (CALDAS, COLOMBIA)*
}

\author{
Paula Lorena Arias-Ortega', Kelly Joahnna Guapacha-Marulanda², Beatriz Toro-Restrepo ${ }^{3}$
}

\begin{abstract}
Resumen
En la reserva de la Central Hidroeléctrica de Caldas (CHEC) se comparó la diversidad y densidad de de macroinvertebrados edáficos de las plantaciones en regeneración de urapán (Fraxinus chinensis) (PU) y eucalipto (Eucalyptus grandis) (PE) y un bosque secundario. Se realizaron dos muestreos entre enero y junio de 2011 al final de la época de lluvias, estableciéndose en cada uno de los sitios tres transectos lineales de $40 \mathrm{~m}$, en los cuales se sacaron cinco monolitos de acuerdo al protocolo del "Tropical Soil Biology and Fertility". Los macroinvertebrados fueron extraídos in situ, se evaluó la estructura vegetal y se hizo análisis de fertilidad del suelo. Se encontró que la mayor densidad de los organismos colectados fue del phylum Arthropoda, dentro del cual la clase Insecta fue la que presentó la mayor $(65,1 \%)$ densidad y la menor fue para Adenophorea del phylum Nematoda (0,3\%). Después de realizar la prueba de Dunnett no se encontraron diferencias significativas en la diversidad $(\mathrm{p}=0,5$ y 0,4$)$ y la densidad $(\mathrm{p}=0,8$ y 0,4$)$ entre las plantaciones. El análisis de componentes principales (ACP) indicó que solo la plantación de eucalipto presentó correlaciones positivas entre la densidad de macroinvertebrados y variables como el área basal y el diámetro a la altura del pecho, y que en los tres sitios la diversidad de Hill se correlacionó positivamente con los contenidos de K, Ca, Mg, M.O y N. Se concluye que ambas plantaciones presentan similar heterogeneidad por lo que se asemejan al bosque secundario, lo que se reflejó en la diversidad y densidad de los macroinvertebrados.
\end{abstract}

Palabras clave: macroinvertebrados edáficos, plantaciones en regeneración, Fraxinus chinensis, Eucalyptus grandis, diversidad, densidad.

\section{INFLUENCE OF THE REGENERATION OF PLANTATION FOREST ON THE DIVERSITY AND DENSITY OF SOIL MACROINVERTEBRATES (CALDAS, COLOMBIA)}

\begin{abstract}
The influence of regenerating plantations of Urapán (PU) and Eucalyptus (PE) was assessed on macroinvertebrate edaphic diversity and density in comparing to secondary forest in the Hydroelectric Caldas (CHEC) Nature Reserve. Two samplings were

\footnotetext{
${ }^{*}$ FR: 26-VIII-2013 . FA: .20-XI-2014

${ }^{1}$ E-mail: paloar13@yahoo.es. Bióloga. Investigadora independiente.

${ }^{2}$ E-mail: kellyjohannaster@hotmail.com. Bióloga. Investigadora independiente.

${ }^{3}$ Profesor asistente, Departamento de Ciencias Biológicas, Universidad de Caldas. Doctor en Ciencias. Manizales,

Colombia. E-mail: beatriz.toro@ucaldas.edu.co
} 
performed at the end of the rainy season. At each site were established three linear transects of $40 \mathrm{~m}$, in which five monoliths were extracted according to protocol of "Tropical Soil Biology and Fertility". The macroinvertebrates were extracted in situ and the same time was evaluated vegetal structure and was analyzed the fertility of soil. The highest density of organisms collected was the phylum Arthropoda, in which the class Insecta was the one with the highest percentage $(65.1 \%)$ and the lowest was Adenophorea the phylum Nematoda (0.3\%). Dunnett's test showed that no significant differences in diversity $(\mathrm{p}=0.5$ and 0.4$)$ and density $(\mathrm{p}=0.8$ and 0.4) between the plantations. Principal Component Analysis (PCA) indicated that only eucalyptus plantation showed a positive correlation between the density of macroinvertebrates and variables such as basal area and diameter at breastheight, and that in all three sites was positively correlated the diversity index of Hill with the contents of $\mathrm{K}, \mathrm{Ca}, \mathrm{Mg}, \mathrm{MO}$ and $\mathrm{N}$. We conclude that both plantations have similarly heterogeneity so resembling secondary forest, which was reflected in the diversity and density of soil macroinvertebrates.

Key words: soil macroinvertebrates, regenerating plantations, Fraxinus chinensis, Eucalyptus grandis, diversity, density.

\section{INTRODUCCIÓN}

El suelo es un sistema vivo y dinámico (JOHNSTON et al., 2004), cuya salud depende de interacciones complejas entre sus propiedades físicas, químicas y biológicas (WILLIAMS et al., 2010), es el sistema clave para el funcionamiento de los ecosistemas terrestres, donde se llevan a cabo dos procesos vitales: la descomposición y el flujo de nutrientes. Estos procesos son controlados principalmente por la actividad biológica, la cual depende en última instancia de la temperatura y la humedad (CHAMORRO-BELLO, 2001; FRAGOSO et al., 2001).

La macrofauna enriquece el suelo con sus deyecciones, excreciones, secreciones y aun con sus propios cadáveres. Permite la destrucción mecánica de los restos vegetales y animales que componen la hojarasca hasta su desmenuzamiento y, consecuentemente, aumentan la superficie expuesta al ataque de las bacterias y microorganismos en general. Contribuyen a la diseminación de esporas fúngicas y otros microrganismos y a la distribución de los sustratos nutritivos, con lo que facilitan la actividad degradadora de la microfauna del suelo. Sus deyecciones ayudan a formar la microestructura del suelo. Airean el suelo, además de permitir una mayor penetración del agua, al construir galerías en él (CABRERA \& CRESPO, 2001). Es probable que la degradación química y física del suelo esté íntimamente relacionada con la disminución de las poblaciones o la pérdida cuantitativa y cualitativa de invertebrados claves de la macrofauna edáfica que regula el ciclo de la materia orgánica y la producción de estructuras físicas biogénicas (DOUBE \& SCHMIDT, 1997; LAVELLE, 2000).

Con frecuencia, el establecimiento de plantaciones forestales generan la fragmentación del bosque nativo (LEÓN-GAMBOA et al., 2010), es perjudicial para la conservación de la biodiversidad, sin embargo, sus efectos netos también dependen 
en gran medida de la cobertura vegetal que haya sido o vaya a ser reemplazada (BROCKERHOFF et al., 2008). Sobre la fauna edáfica puede causar efectos directos e indirectos, afectar la mineralización y humificación de materia orgánica, la cual determina en gran medida el estado de los nutrientes en el suelo. Los efectos directos incluyen la calidad y cantidad de hojarasca, los indirectos incluyen cambios en las condiciones microclimáticas como la temperatura, la humedad y el hábitat físico (WARREN \& ZOU, 2002). La introducción de especies exóticas (plantaciones forestales y especies invasoras del suelo) puede traer graves consecuencias para la biodiversidad del suelo porque puede alterar los componentes bióticos y abióticos del medio, y modificar las interacciones competitivas dentro de las comunidades (DECAËNS et al., 2006). Por otro lado, las plantaciones pueden facilitar la sucesión de los sotobosques en sitios degradados a través de la modificación de condiciones microclimáticas, estimulando la acumulación de carbono orgánico en las capas de la superficie del suelo e incrementando la complejidad estructural (PARROTTA et al., 1997).

En Colombia, las plantaciones forestales han sido utilizadas desde la segunda mitad del siglo XX para la protección de cuencas asociadas al manejo del recurso hídrico (CASTAÑO-VILLA et al., 2008). Dichos agroecosistemas comenzaron a ser evaluados en torno a su posible función como catalizadores para la recuperación de áreas degradadas a finales del siglo pasado (PARROTTA et al., 1997). En el caso de la reserva forestal protectora de la Central Hidroeléctrica de Caldas (CHEC), el proceso de reforestación con fines de protección de la zona dedicada a la conservación y la regulación del agua, se inició en 1962 y se estableció en las áreas de pastizales e incluyó 20 especies con las que se cubrieron 66,8 ha, entre 2300 y $2700 \mathrm{~m}$, dentro de las que se incluyen especies exóticas como Eucalyptus grandis y Fraxinus chinensis (ÁLVAREZ-MEJÍA et al., 2007).

Esta investigación se realizó con el fin de evaluar la influencia que tienen la plantación forestal de urapán (PU) y de eucalipto (PE) sobre la diversidad y densidad de macroinvertebrados edáficos en la reserva de la CHEC, por lo cual se determinó la relación de la densidad de macroinvertebrados con la estructura vegetal y algunas variables ambientales, y se evaluaron algunos aspectos fisicoquímicos del suelo y su relación con la diversidad de los mismos.

\section{MATERIALES Y MÉTODOS}

\section{Sitio de estudio}

La reserva forestal protectora de la Central Hidroeléctrica de Caldas (Figura 1), se encuentra en el flanco sur de la vertiente alta del río Chinchiná (ÁLVAREZ-MEJíA et al., 2007), en jurisdicción de los municipios de Manizales y Villamaría, del departamento de Caldas. Limita al norte con el río Chinchiná y al sur con el río Molinos ( $5^{\circ} 1^{\prime} \mathrm{N}-75^{\circ} 24^{\prime} \mathrm{O}$ ). Comprende unas 3892 ha de bosque protegido, desde los 2400 hasta 3400 msnm (ÁLVAREZ-MEJÍA et al., 2007), presenta un rango de temperatura entre los 12 y $18^{\circ} \mathrm{C}$, y una precipitación promedio anual entre los 1000 a $2000 \mathrm{~mm}$ (GIRALDO, ined.). HOLDRIDGE (1967) clasifica esta zona de vida como bosque húmedo montano bajo (bh-MB). Según ÁLVAREZ-MEJÍA et al. (2007) en esta reserva se presentan espacios donde el dosel arbóreo es uniespecífico, con especies cultivadas como aliso (Alnus acuminata), urapán (F. chinensis), ciprés 
(Cupressus lusitánica), pino (Pinus spp.) y eucalipto (Eucalyptus spp.). Además, los sitios de estudio que se escogieron para esta investigación llevan un proceso de regeneración desde 1970 (ANÓNIMO, ined.; GIRALDO, ined.).

Uno de los sitios de estudio, el bosque secundario (BS), está localizado a $5^{\circ} 1^{\prime} \mathrm{N}$ $75^{\circ} 42^{\prime} \mathrm{O}$, a una altitud promedio de $2454 \mathrm{msnm}$ en un terreno escarpado con una pendiente superior al 75\%. Presenta frecuentes sucesos de perturbación causados por dicha pendiente, la elevada precipitación y factores antrópicos. A los otros sitios, la plantación de urapán (PU) y de eucalipto (PE), se les realizaron entresacas a los 30 años de edad para fines de conservación y se extrajeron solo los árboles con problemas fitosanitarios, de deformidad y muy inclinados. La plantación de urapán tiene 21,3 ha y presenta una densidad de 720 árboles por hectárea. Ambas plantaciones se encuentran localizadas a $5^{\circ} 2^{\prime} \mathrm{N}-75^{\circ} 42^{\prime} \mathrm{O}$, a una altitud promedio entre 2445 y 2426 msnm (GIRALDO, ined.).

\section{Mapa de localización}
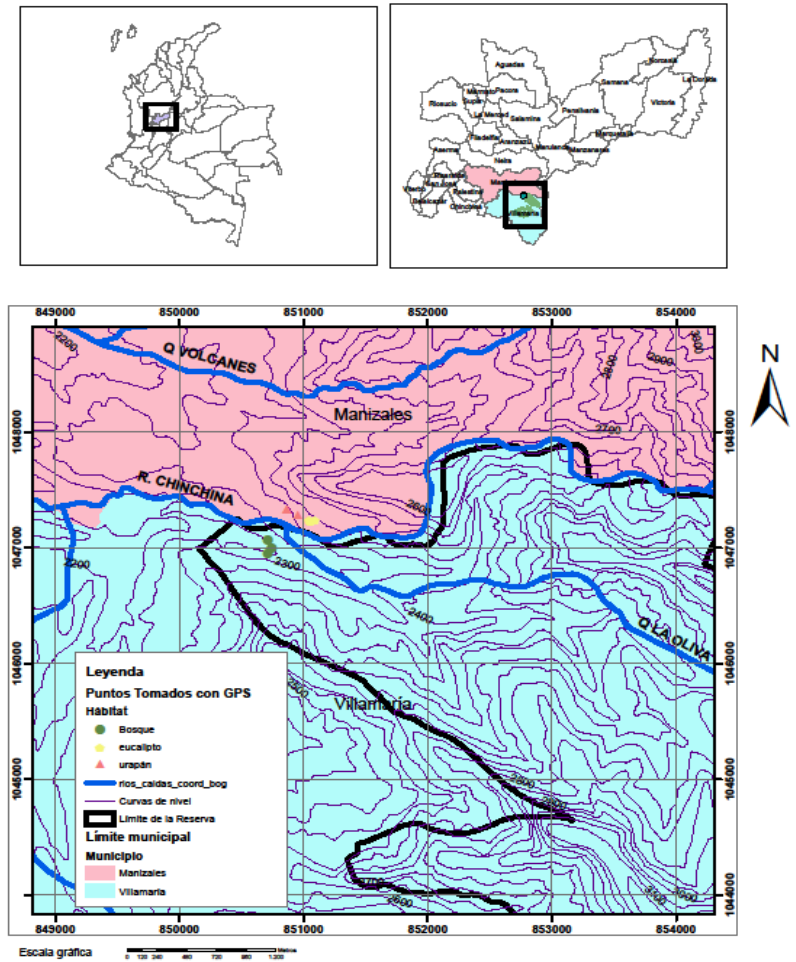

Figura 1. Mapa de localización de la reserva forestal protectora de la Central Hidroeléctrica de Caldas (CHEC). 


\section{Toma de datos}

Se realizaron dos muestreos (24 enero-17 junio de 2011), en época de bajas lluvias cuando existe mayor actividad de los macroinvertebrados (HUERTA-LWANGA et al., 2008), estableciéndose tres transectos lineales de $40 \mathrm{~m}$ en los diferentes sitios de estudio. En cada transecto se extrajeron cinco monolitos de suelo de 25x25x30 cm de acuerdo a la metodología del TSBF (ANDERSON \& INGRAM, 1993), separados $10 \mathrm{~m}$ entre sí, divididos en cuatro estratos: 0+ (mulch), 0-10, 10-20 y de 20-30 cm de profundidad. In situ se fijaron las lombrices en formol al 5\% y los individuos restantes en alcohol al 70\% (ZALDÍVAR-SUÁREZ et al., 2009). En el caso de las lombrices fue necesario utilizar un protocolo de fijación y conservación más específico para facilitar su identificación (MORENO, 2004), y en el de los pseudoescorpiones se hizo un montaje con placas excavadas en el laboratorio (ADIS, 2002; HARVEY, 1992).

Antes de realizar la extracción de los monolitos, en parcelas circulares de $50 \mathrm{~m}^{2}$ (radio $4 \mathrm{~m}$ ) se midieron los siguientes parámetros estructurales de la vegetación (AUGUST, 1983): 1) número de estratos, 2) altura de dosel, 3) altura de las herbáceas, 4) porcentaje de la cobertura de dosel, 5) porcentaje de cobertura de herbáceas, 6) porcentaje de la cobertura horizontal, 7) área basal y 8) individuos con un DAP $\geq 1 \mathrm{~cm}$. Se utilizó el método de cuadrantes para medir la cobertura horizontal (ETIENNE \& PRADO, 1982; JARAMILLO, ined.) y el protocolo de biometría (ANÓNIMO, 2003). Posterior a la extracción de cada monolito, se tomaron $100 \mathrm{~g}$ de suelo para determinar su humedad y $1 \mathrm{~kg}$ para el análisis de fertilidad (Tabla 1) que se realizó en el en el laboratorio de Multilab Agroanalítica del Centro Nacional de Investigaciones de Café (Cenicafé).

Tabla 1. Método del análisis de fertilidad de suelos.

\begin{tabular}{ll}
\hline Variables fisicoquímicas & Método de análisis \\
\hline $\mathrm{pH}$ & Potenciométrico - suelo: Agua 1:1 \\
$\mathrm{N}$ & Calculado; M.O: Walkley-Black - Colorimetría \\
$\mathrm{Ca}, \mathrm{Mg}$ & Acetato de amonio 1N pH 7,0-EAA \\
$\mathrm{Al}$ & KCI IM-EAA \\
$\mathrm{P}$ & Bray II - colorimetría Bray Kurtz \\
\multirow{2}{*}{ Textura } & Bouyoucos con pirofosfato de sodio - clasificación diagrama triangular \\
& de USDA - F (franco), Ar (arcilloso), L (limoso), A (arenoso) \\
\hline
\end{tabular}

\section{Trabajo de laboratorio}

Los individuos encontrados fueron identificados hasta el nivel taxonómico de familia. Se emplearon las claves taxonómicas de STEHR $(1987,1991)$ para la identificación de los insectos inmaduros, las de TRIPLEHORN \& JOHNSON (2005), las recopiladas por ARNETT \& THOMAS (2001), ARNETT et al. (2002) para los insectos adultos, y por ADIS (2002) para identificar los miriápodos, las de CHAMORROBELLO (ined.) para las lombrices (adaptadas de STRESEMANN, 1986), las de JOCQUÉ \& DIPPENAAR-SCHOEMAN (2007) para las arañas, y las de HARVEY (1992) para la determinación de los pseudoescorpiones. Se clasificaron en grandes grupos como 
diplópodos, insectos sociales (hormigas), lombrices de tierra, larvas de insectos y otros, de acuerdo a LORANGER-MERCIRIS et al. (2007).

\section{Análisis de los datos}

Se calculó la densidad de macroinvertebrados del suelo encontrados en los monolitos, y se estimó su diversidad mediante el índice ecológico de HILL (1973), el cual integra el índice de Shannon y el de Simpson (MORENO, 2001). Se aplicó la prueba de Shapiro-Wilk y la prueba de Levene de igualdad de las varianzas. Como los datos de la densidad y diversidad de Hill no presentaron una homogeneidad de varianza se transformaron mediante la ecuación x' = Ln (x), sumándole uno a los valores originales de la diversidad, ya que fueron menores a dicho número: $\mathrm{x}^{\prime}=\mathrm{Ln}$ $(\mathrm{x}+1)$. Después de transformarlos se realizó la prueba paramétrica de Dunnett con el software SPSS versión 17, para comparar el bosque secundario (sitio control) con las plantaciones forestales, y se realizó un análisis de componentes principales (ACP) con el software SPAD versión 7.3 para determinar si los parámetros estructurales de la vegetación y las variables ambientales evaluadas estaban relacionados con la densidad de macroinvertebrados edáficos y cuáles propiedades fisicoquímicas del suelo estaban relacionadas con la diversidad de la macrofauna en los sitios. Se calculó el índice de Margalef (MORENO, 2001) para determinar la diversidad de especies vegetales en los sitios, y los índices de complejidad y heterogeneidad se hallaron respectivamente a partir de la media y de la desviación estándar obtenidas del primer componente (PCI) que arroja el ACP (AUGUST, 1983). Además, se realizaron pruebas paramétricas (ANOVAs) y no paramétricas (Kruskal-Wallis) para comparar las variables fisicoquímicas del suelo entre los sitios.

\section{RESULTADOS Y DISCUSIÓN}

\section{Propiedades del suelo y variables ambientales}

Los resultados de los análisis fisicoquímicos del suelo indican que las plantaciones son similares entre sí y se aproximan a las características del bosque secundario excepto en la textura del suelo, ya que el bosque secundario presenta más texturas (franco-arenoso, franco, franco-arcilloso) y porcentajes más altos (Tabla 2).

En cuanto a los elementos, tres de los cinco evaluados estuvieron en mayor concentración en la plantación de urapán ( $\mathrm{N}=0,4 ; \mathrm{Ca}=7,6 ; \mathrm{Mg}=1,3)$, lo que puede favorecer la fertilidad del suelo, mientras que en el bosque secundario y la plantación de eucalipto estos elementos se presentaron en menores concentraciones. La plantación de urapán presentó mayor porcentaje promedio de materia orgánica (11), lo que puede indicar que los materiales orgánicos aportados al suelo, que dependen sensiblemente del tipo de cobertura vegetal y la velocidad con la cual estos se descomponen, exhiben mayor actividad (ZAPATA \& OSORIO, 2010) en comparación con los otros sitios. 
Tabla 2. Valores promedio de la fertilidad del suelo en los tres sitios de muestreo.

\begin{tabular}{|c|c|c|c|c|}
\hline Fisicoquímicos & & PU & PE & BS \\
\hline $\mathrm{pH}$ & & 5,9 & 5,4 & 5,1 \\
\hline \multirow[t]{2}{*}{ M.O (\%) } & & 11 & 8,2 & 7 \\
\hline & $\mathrm{N}^{*}$ & 0,4 & 0,3 & 0,3 \\
\hline \multirow[t]{3}{*}{ Macronutrientes } & $\mathrm{P}^{* *}$ & 28,5 & 33,2 & 20 \\
\hline & $\mathrm{K}^{* * *}$ & 0,2 & 0,2 & 0,2 \\
\hline & $\mathrm{Ca}^{* * *}$ & 7,6 & 2,9 & 3,2 \\
\hline \multirow[t]{3}{*}{ Micronutrientes } & $\mathrm{Mg}^{* * *}$ & 1,3 & 0,8 & 1,2 \\
\hline & $\mathrm{K}^{* * *}$ & 0,2 & 0,2 & 0,2 \\
\hline & $\mathrm{Al}^{* * *}$ & 0,3 E-1 & 0,3 & 1,5 \\
\hline \multirow[t]{2}{*}{ Humedad (\%) } & & 35,4 & 30,5 & 34,1 \\
\hline & Arcillas* & 8,4 & 8,3 & 20,1 \\
\hline \multirow[t]{3}{*}{ Textura } & Limos* & 18,3 & 15,8 & 28,4 \\
\hline & Arenas* & 73,3 & 76,1 & 51,5 \\
\hline & & F.A & F.A & F.A, F, F.Ar \\
\hline
\end{tabular}

* (\%), ${ }^{* *}\left(\mathrm{mg} \cdot \mathrm{kg}^{-1}\right),{ }^{* * *}\left(\mathrm{cmol}(+) \cdot \mathrm{kg}^{-1}\right)$, PU: Plantación de urapán, PE: Plantación de eucalipto, BS: Bosque secundario, M.O: Materia orgánica, F.A: Franco-arenoso, F: Franco, F.Ar: Franco-arcilloso.

\section{Estructura y composición vegetal}

La composición vegetal fue similar en los tres sitios, encontrándose el mayor número de especies en la plantación de urapán con 49 especies (Tabla 3). En la plantación en regeneración de urapán (F. chinensis) representó el 9,8\% de los individuos encontrados y sigue siendo la especie dominante con un $83 \%$. En la plantación en regeneración de eucalipto el 4,2\% de los individuos son de E. grandis, sin embargo, esta especie representa el $58 \%$ de la dominancia.

En los tres sitios, la especie más abundante fue la palma Chamaedorea linearis, con un porcentaje entre 24 y 29\%, lo que indica un buen estado de regeneración. Según MENDOZA \& OYAMA (1999), C. linearis posee una enorme capacidad de adaptación a las perturbaciones y transformaciones de su hábitat, lo cual le ha permitido pasar a través de fuertes cambios climáticos así como poder adaptarse a diferentes condiciones, en situaciones ambientales muy diversas.

En las dos plantaciones en regeneración las especies más importantes ecológicamente siguen siendo el urapán con un 32\% y el eucalipto con un 22\%, sin embargo, en la estructura y composición de la regeneración natural que se lleva a cabo en su interior presentan algunas similitudes, incluso con el bosque secundario. Especies como C. linearis, Hedyosmum bonplandianum y Oreopanax floribundum estuvieron entre las cinco más importantes ecológicamente en los tres sitios. La similitud de estas especies, que a su vez fueron las más abundantes, sugiere de alguna manera, que el proceso de sucesión natural que tiene lugar en los dos tipos de plantaciones 
se está aproximando a la del bosque secundario. Esto se ratifica en que los sitios presentan una heterogeneidad similar (Tabla 4). En los tres se registró el mismo número promedio de estratos, pero el porcentaje promedio de cobertura de dosel fue mayor en el bosque y urapán ( $83 \%$ y 80\%, respectivamente) que en la plantación de eucalipto (73\%).

Tabla 3. Composición de la vegetación en los tres sitios.

\begin{tabular}{lccccc}
\hline Hábitat & $\begin{array}{c}\text { No. } \\
\text { individuos }\end{array}$ & No. especies & $\begin{array}{c}\text { Área basal } \\
(\mathbf{m})\end{array}$ & ${ }^{*}$ Diversidad & $\begin{array}{c}\text { Área } \\
\left(\mathbf{m}^{2}\right)\end{array}$ \\
\hline PU & 599 & 49 & 5 & 7.5 & 750 \\
PE & 332 & 37 & 4,3 & 6.2 & 750 \\
BS & 264 & 47 & 2.5 & 8.3 & 750 \\
Total & 1195 & 78 & 11,8 & ---- & 2250 \\
\hline
\end{tabular}

PU: Plantación de urapán, PE: Plantación de eucalipto, BS: Bosque secundario, * Índice de Margalef (MORENO, 2001).

Tabla 4. Valores de los índices de complejidad y heterogeneidad del hábitat, derivados del análisis de componentes principales del bosque secundario (BS), la plantación de urapán (PU) y eucalipto $(\mathrm{PE})$, obtenido a partir de las variables estructurales de la vegetación.

\begin{tabular}{lccc}
\hline Índice & PU & PE & BS \\
\hline Complejidad & 0,3 & $-0,1 \mathrm{E}-1$ & 0,3 \\
Heterogeneidad & 0,6 & 0,6 & 0,5 \\
\hline
\end{tabular}

\section{Macroinvertebrados del suelo}

Al comparar el bosque secundario con las dos plantaciones, la diversidad y densidad de macroinvertebrados edáficos no presentaron diferencias significativas $(\mathrm{p}>$ $0,05)$, lo que indica que las familias de macroinvertebrados son muy similares en los sitios (Tabla 5 y Tabla 6).

Tabla 5. Prueba de Dunnett para comparar la diversidad de Hill entre los sitios. Intervalo de confianza del $95 \%$.

\begin{tabular}{ccccccc}
\hline (I) Hábitat & $\begin{array}{c}\text { (J) } \\
\text { Hábitat }\end{array}$ & $\begin{array}{c}\text { Diferencia } \\
\text { de medias } \\
(\mathrm{I}-\mathrm{J})\end{array}$ & $\begin{array}{c}\text { Error } \\
\text { estándar }\end{array}$ & $\begin{array}{c}\mathrm{p} \\
\text { (significancia) }\end{array}$ & $\begin{array}{c}\text { Límite } \\
\text { inferior }\end{array}$ & $\begin{array}{c}\text { Límite supe- } \\
\text { rior }\end{array}$ \\
\hline PU & BS & $3 \mathrm{E}-2$ & $3 \mathrm{E}-2$ & $\mathbf{0 , 5}$ & $-4 \mathrm{E}-2$ & $9,7 \mathrm{E}-2$ \\
$\mathrm{PE}$ & $\mathrm{BS}$ & $-3 \mathrm{E}-2$ & $3 \mathrm{E}-2$ & $\mathbf{0 , 4}$ & $-0,1$ & $3 \mathrm{E}-2$ \\
\hline
\end{tabular}


Tabla 6. Prueba de Dunnett para comparar la densidad entre los sitios. Intervalo de confianza del 95\%.

\begin{tabular}{ccccccc}
\hline (I) Hábitat & (J) Hábitat & $\begin{array}{c}\text { Diferencia } \\
\text { de medias } \\
\text { (I-J) }\end{array}$ & $\begin{array}{c}\text { Error } \\
\text { estándar }\end{array}$ & $\begin{array}{c}\mathrm{p} \\
\text { (significancia) }\end{array}$ & $\begin{array}{c}\text { Límite } \\
\text { inferior }\end{array}$ & $\begin{array}{c}\text { Límite } \\
\text { superior }\end{array}$ \\
\hline PU & BS & 0,1 & 0,2 & $\mathbf{0 , 8}$ & $-0,4$ & 0,6 \\
PE & BS & 0,3 & 0,2 & $\mathbf{0 , 4}$ & $-0,3$ & 0,8 \\
\hline
\end{tabular}

Se registró una densidad promedio de 312,3 ind $/ \mathrm{m}^{2}$, la mayoría en estado inmaduro, de los phylum Arthropoda, Annelida y Nematoda, identificándose 25 órdenes y 116 familias. La mayor densidad fue del phylum Arthropoda, dentro del cual la clase Insecta fue la que presentó mayor densidad $(65,1 \%)$, seguida de la clase Diplopoda (23,5\%), Chilopoda (6,1\%), Malacostraca (3,3\%), Arachnida (1,3\%); Clitellata del phylum Annelida $(0,4 \%)$ y Adenophorea del phylum Nematoda $(0,3 \%)$ fueron las que presentaron menor densidad.

En un área de $2250 \mathrm{~m}^{2}$, ambas plantaciones presentaron la mayor densidad y número de familias de macroinvertebrados edáficos. La plantación de eucalipto registró un total de 1481,1 ind $/ \mathrm{m}^{2}$ y 86 familias, la plantación de urapán un total de 1200,5 ind $/ \mathrm{m}^{2}$ y 78 familias, mientras que el bosque secundario presentó 1066,1 ind $/ \mathrm{m}^{2}$ y 74 familias.

Algunos órdenes como Diptera presentaron mayor densidad en la plantación de eucalipto que en la plantación de urapán y el bosque secundario, otros como Hymenoptera y Polydesmida fueron más representativos en el bosque secundario y en la plantación de urapán, respectivamente (Figura 2).

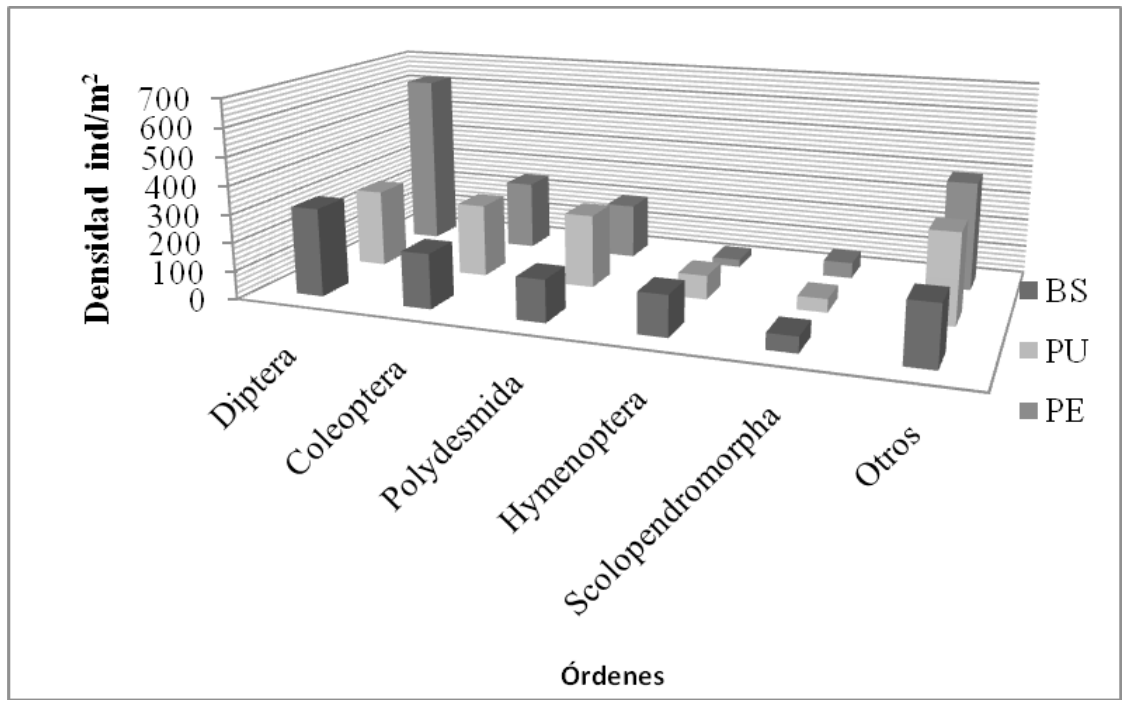

Figura 2. Densidad de macroinvertebrados edáficos de los órdenes más representativos identificados en el bosque secundario (BS), la plantación de urapán (PU) y eucalipto (PE). 
Las larvas de insectos fueron las que presentaron mayor densidad en los tres sitios (Tabla 7), siendo las más representativas las larvas de dípteros en la plantación de eucalipto $\left(619,7 \mathrm{ind} / \mathrm{m}^{2}\right)$. En las plantaciones de urapán y eucalipto las familias de dípteros más abundantes fueron Chironomidae, Ceratopogonidae y Scatopsidae, mientras que en el bosque secundario fueron Tipulidae y Sciaridae.

Tabla 7. Densidad de macroinvertebrados edáficos, clasificados en grandes grupos, encontrados en dos plantaciones: urapán (PU), eucalipto (PE) y en un bosque secundario (BS).

\begin{tabular}{|c|c|c|c|}
\hline \multirow{2}{*}{ Grupo } & \multicolumn{3}{|c|}{ Sitio $\left(\right.$ ind $\left./ \mathbf{m}^{2}\right)$} \\
\hline & PU & PE & BS \\
\hline Diplópodos & 353,1 & 315,7 & 210,1 \\
\hline Insectos sociales (hormigas) & 85,3 & 26,7 & 143 \\
\hline Lombrices & 6,4 & 5,9 & 2,1 \\
\hline Larvas de insectos & 542,4 & 855,5 & 524,8 \\
\hline Otros & 213,3 & 277,3 & 186,1 \\
\hline Total & 1200,5 & 1481,1 & 1066,1 \\
\hline
\end{tabular}

La presencia de dípteros en estado inmaduro concuerda con lo encontrado por HERNÁNDEZ-ORTIZ \& DZUL-CAUICH (2008), quienes sugieren que el incremento del régimen de lluvias y la temperatura media favorecen su establecimiento, lo que se ve vio reflejado en el presente estudio en la plantación de eucalipto debido a la presencia de charcos y posiblemente a las condiciones microclimáticas que ofrece la plantación. Si se excluyeran los dípteros de los resultados obtenidos, la plantación de urapán presentaría mayor cantidad de individuos en comparación con los otros sitios (Tabla 8), lo que estaría acorde con los resultados obtenidos del análisis de fertilidad del suelo.

Tabla 8. Abundancia y número de familias de dípteros en el bosque secundario (BS), la plantación de urapán (PU) y eucalipto (PE).

\begin{tabular}{lc|c|c|ccc}
\hline Sitio & \multicolumn{3}{c}{ Organismos } & \multicolumn{2}{c}{ Total } & Total \\
& \multicolumn{2}{c}{ Dípteros } & \multicolumn{2}{c}{ Otros } & A & F \\
& A & F & A & F & & 78 \\
\hline PU & 519 & 19 & 1732 & 59 & 2251 & 86 \\
PE & $\mathbf{1 1 7 0}$ & 16 & 1607 & 70 & 2777 & 74 \\
BS & 586 & 16 & 1413 & 58 & 1999 & \\
\hline
\end{tabular}

A: Abundancia, F: Número de familias.

Si se tienen en cuenta los resultados de la prueba de Dunnett y del índice de heterogeneidad, se puede deducir que la variación horizontal en la fisionomía de los tres hábitats influye en la densidad de macroinvertebrados edáficos, y que a su vez la heterogeneidad del hábitat puede ser influenciada por la arquitectura de las plantaciones, las condiciones microclimáticas que generan en el sotobosque (PARROTTA et al., 1997), como variación de la intensidad lumínica que aumenta con la formación de claros, y la calidad de su hojarasca que depende de la mezcla de diferentes tipos, e influye en la actividad microbiana y de la fauna, por ende se ve afectada la manera en que la hojarasca se descompone (IRMLER, 2000). 
Con respecto al índice de diversidad de Hill, se encontraron valores que oscilan entre 0,4 y 0,9 (Tabla 9). Estos resultados indican que las familias menos abundantes, con un rango de tolerancia más restringido, están mejor representadas que las más abundantes (MORALES \& SARMIENTO, 2002), lo que sugiere que los sitios ya no se encuentran muy perturbados, partiendo de la base de que se encuentran en sucesión secundaria y su proceso de regeneración comenzó desde 1970.

Tabla 9. Diversidad de la macrofauna edáfica en dos plantaciones: urapán (PU), eucalipto (PE) y en un bosque secundario (BS).

\begin{tabular}{lccccccc}
\hline Sitio & $\begin{array}{c}\text { Estrato } \\
(\mathbf{c m})\end{array}$ & Clases & Órdenes & Familias & N1 & N2 & E' \\
\hline PU & $0+$ & 6 & 17 & 56 & 15,1 & 9,2 & 0,6 \\
& $0-10$ & 7 & 20 & 60 & 20,1 & 13,6 & 0,7 \\
& $10-20$ & 5 & 12 & 27 & 15,1 & 11,5 & 0,8 \\
& $20-30$ & 5 & 12 & 22 & 12,1 & 8,6 & 0,7 \\
PE & $0+$ & 5 & 18 & 57 & 9,2 & 4,1 & 0,4 \\
& $0-10$ & 7 & 21 & 67 & 23,2 & 15,4 & 0,7 \\
& $10-20$ & 6 & 14 & 28 & 17,2 & 11,7 & 0,7 \\
& $20-30$ & 4 & 10 & 16 & 12,9 & 10,4 & 0,8 \\
& $0+$ & 5 & 19 & 55 & 14,7 & 8,3 & 0,6 \\
& $0-10$ & 7 & 21 & 51 & 17 & 9,2 & 0,5 \\
& $10-20$ & 7 & 15 & 24 & 15,7 & 12 & 0,8 \\
& $20-30$ & 4 & 10 & 14 & 12 & 10,5 & 0,9 \\
& 4 & 7 & 25 & 118 & $-\ldots--$ & $-\ldots--$ & $-\cdots$ \\
\hline
\end{tabular}

N1 y N2: Serie de números de Hill, E’: Índice de equidad de Hill (N2/N1).

Los dos primeros factores (ejes) del análisis de componentes principales que correlacionaron la diversidad de Hill con las variables fisicoquímicas explican el 65,1\% de la variación. El vector de la diversidad de Hill en este caso fue poco representativo (Figura 3), posiblemente porque solo se pudo llegar al nivel taxonómico de familia, e indica que la diversidad de Hill en los tres sitios está relacionada positivamente con los contenidos de $\mathrm{K}, \mathrm{Ca}, \mathrm{Mg}, \mathrm{M} . \mathrm{O}$, y N, por tanto se puede deducir que las propiedades del suelo influencian, principalmente el pH, y la diversidad de macroinvertebrados edáficos, tal como lo demostraron LORANGERMERCIRIS et al. (2007). 


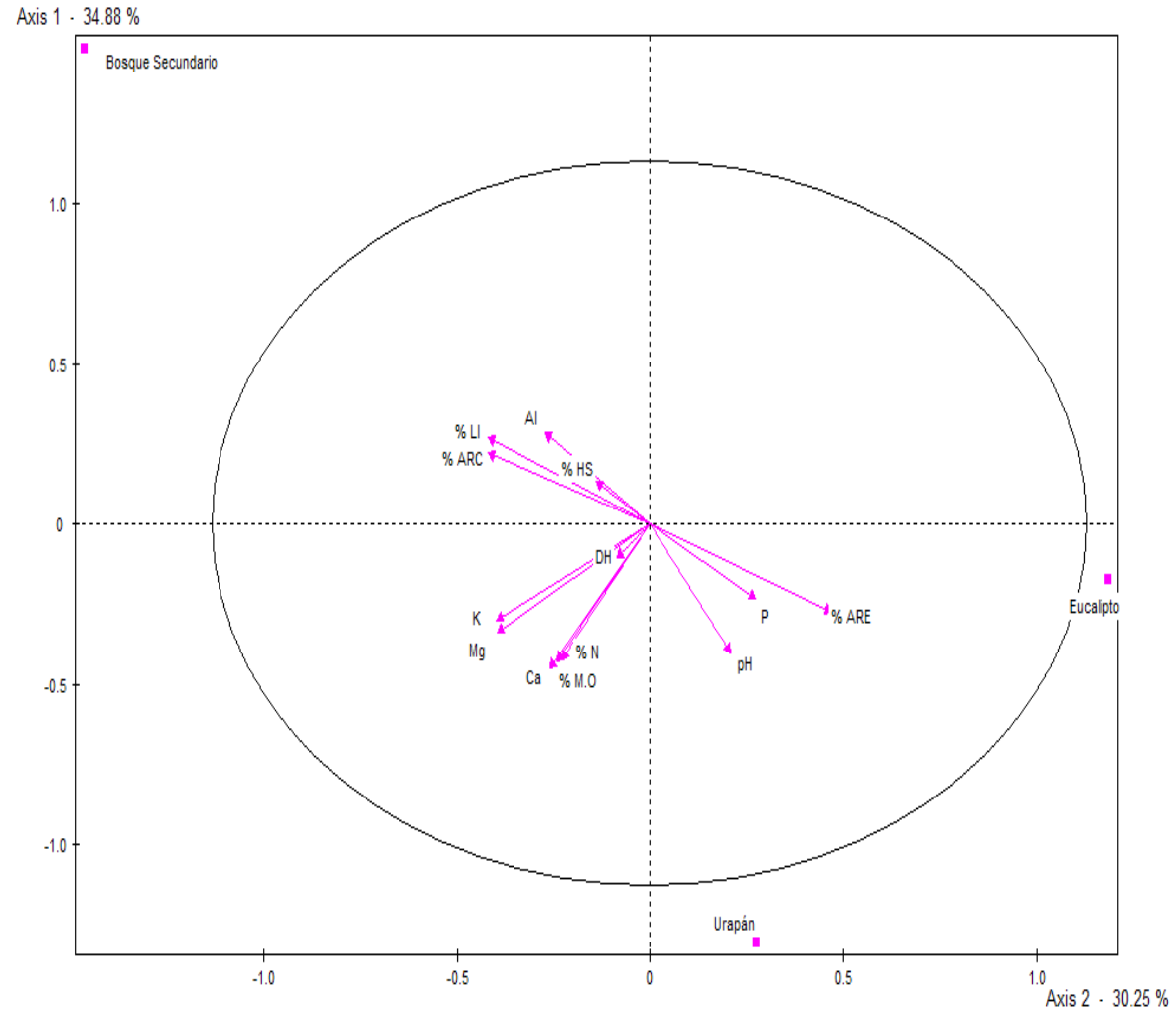

Figura 3. Círculo de correlación: diversidad de Hill y variables fisicoquímicas del suelo, resultante del análisis de componentes principales. DH: Diversidad de Hill, Al: Aluminio, \% Li: Porcentaje de limos, \% ARC: Porcentaje de arcillas, \% ARE: Porcentaje de arenas, \% $\mathrm{N}$ : Porcentaje de nitrógeno, \% HS: Porcentaje de humedad del suelo, pH: Potencial de hidrógeno, \% M.O: Porcentaje de materia orgánica, K: Potasio, Mg: Magnesio, Ca: Calcio, P: Fósforo.

Por otro lado, el análisis de componentes principales con un 37,1\% de inercia entre sus ejes (Figura 4) indicó que la densidad de macroinvertebrados edáficos en la plantación de eucalipto se encuentra relacionada positivamente con variables como el área basal, el diámetro a la altura del pecho, la altura total y número de estratos. 


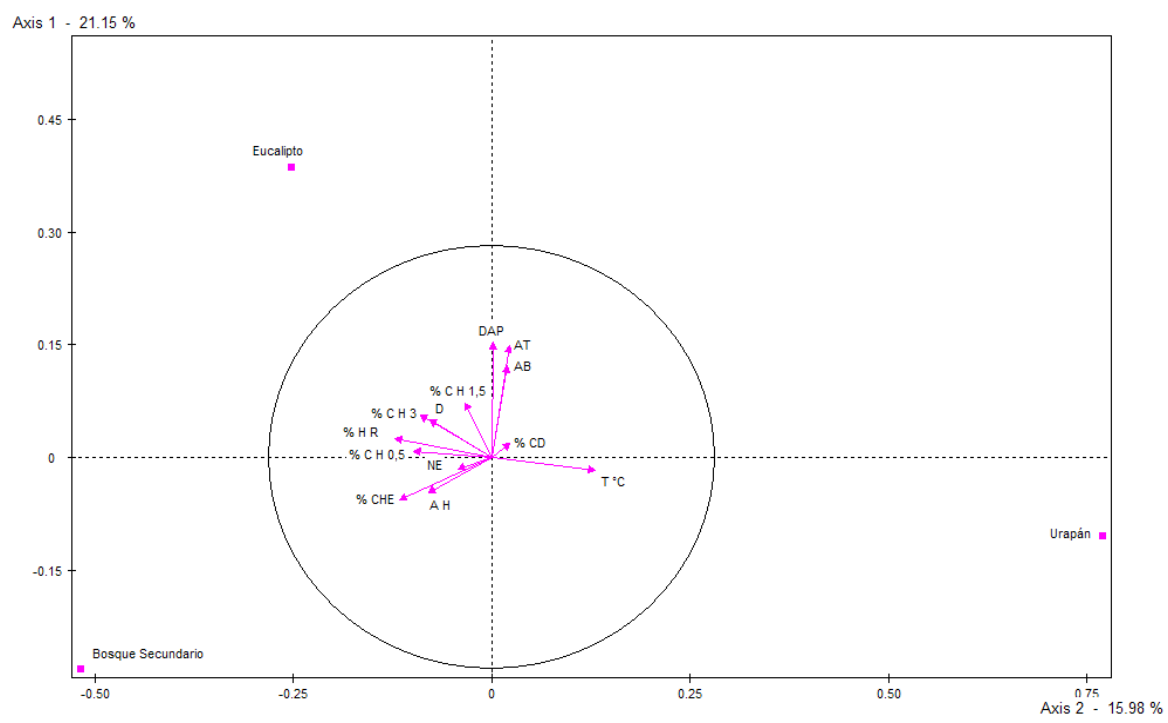

Figura 4. Círculo de correlación: densidad de macroinvertebrados edáficos, variables estructurales de la vegetación y ambientales, resultante del análisis de componentes principales. D: Densidad, \% CHE: Porcentaje de cobertura de herbáceas, DAP: Diámetro a la altura del pecho, AT: Altura total, AB: Área basal, \% CD: Porcentaje de cobertura de dosel, NE: Número de estratos, AH: Altura de herbáceas, \% $\mathrm{CH}$ : Porcentaje de cobertura horizontal (a $0,5,1,5$ y $3 \mathrm{~m}), \%$ HR: Porcentaje de humedad relativa, $\mathrm{T}^{\circ} \mathrm{C}$ : Temperatura ambiental.

Debido a lo anterior, se evidencia que aquellos ambientes con una mayor heterogeneidad espacial contienen más especies, precisamente porque proporcionan una mayor variedad de microhábitats y una gama amplia de microclimas, donde según BEGÓN et al. (2006) la correlación entre la riqueza de especies animales y la diversidad estructural de plantas es marcadamente más intensa que la correlación con la diversidad de especies vegetales. Además, en los ecosistemas terrestres las plantas altas son cuantitativamente los productores primarios más importantes (MANLAY et al., 2002) que poseen DAP mayor, y el dosel de los árboles actúa como recurso para los organismos que pueden colonizar el suelo gracias a que pueden habitar ambos (DE GOEDE, 1996).

El análisis de varianza que se hizo para comparar las variables fisicoquímicas del suelo estimadas, indica que el contenido de materia orgánica (M.O) y N no presentan diferencias significativas entre los sitios (Tabla 10), lo que coincide con que la diversidad de Hill está correlacionada positivamente con estas dos variables en los tres sitios, lo que sugiere que la M.O y el N son propiedades que influyen en la diversidad de macroinvertebrados edáficos de la reserva forestal protectora de la CHEC, además, se asume que el ciclado de nutrientes en las plantaciones y en el bosque secundario es similar. 
Tabla 10. Valores p de las variables estimadas en los tres sitios: plantación de urapán (PU), plantación de eucalipto (PE) y bosque secundario (BS). Nivel de confianza del $95 \%$.

\begin{tabular}{|c|c|c|c|c|c|c|c|}
\hline Variable & S-W* & $\mathbf{B}^{* *}$ & ANOVA & $\mathbf{K}-\mathbf{W}^{* * *}$ & PU-PE & PU-BS & PE-BS \\
\hline$\% \mathrm{HS}$ & $8,5 \mathrm{E}-2$ & 0,3 & 1 E-4 & & $\neq$ & $=$ & $\neq$ \\
\hline $\mathrm{pH}$ & $4,5 \mathrm{E}-2$ & & & 0,5 E-11 & $\neq$ & $\neq$ & $=$ \\
\hline$\%$ ARC & 0 & & & $1,2 \mathrm{E}-9$ & $=$ & $\neq$ & $\neq$ \\
\hline$\%$ LI & 0 & & & $1,1 \mathrm{E}-7$ & $=$ & $\neq$ & $\neq$ \\
\hline$\%$ ARE & 0 & & & $3,0 \mathrm{E}-9$ & $=$ & $\neq$ & $\neq$ \\
\hline$\% \mathrm{~N}$ & $2,5 \mathrm{E}-5$ & & & 8,7 E -2 & $=$ & $=$ & $=$ \\
\hline \% M.O & 1,8 E-3 & & & $6,2 \mathrm{E}-2$ & $=$ & $=$ & $=$ \\
\hline K & 1,0 E-13 & & & 0,45 & $=$ & $=$ & $=$ \\
\hline $\mathrm{Ca}$ & 0 & & & 1,7 E-2 & $=$ & $\neq$ & $=$ \\
\hline $\mathrm{Mg}$ & $2,2 \mathrm{E}-16$ & & & $1,8 \mathrm{E}-2$ & $=$ & $=$ & $\neq$ \\
\hline $\mathrm{Al}$ & 0 & & & $3,8 \mathrm{E}-6$ & $=$ & $\neq$ & $\neq$ \\
\hline $\mathrm{P}$ & $2,1 \mathrm{E}-7$ & & & $4,0 \mathrm{E}-3$ & $=$ & $\neq$ & $\neq$ \\
\hline
\end{tabular}

* Shapiro-Wilk, ${ }^{* *}$ Bartlett, ${ }^{* * *}$ Kruskal-Wallis, \% HS: Porcentaje de humedad del suelo, pH: Potencial de hidrógeno, $\%$ ARC: Porcentaje de arcillas, \% Li: Porcentaje de limos, \% ARE: Porcentaje de arenas, \% N: Porcentaje de nitrógeno, \% M.O: Porcentaje de materia orgánica, K: Potasio, Ca: Calcio, Mg: Magnesio, Al: Aluminio, P: Fósforo.

Si bien, la plantación de eucalipto es la que presentó menor diversidad vegetal y menor complejidad del hábitat, es la que presentó mayor densidad de macroinvertebrados edáficos y es la que tiene correlación positiva entre la densidad de los mismos y las variables estructurales de la vegetación, es posible que el tipo de vegetación de este sitio ofrezca mejores recursos en alimentación, espacio y reproducción de los organismos del suelo.

\section{CONCLUSIONES}

A partir de los resultados obtenidos y de las condiciones en que se desarrolló esta investigación se puede concluir que:

- La densidad y diversidad de macroinvertebrados edáficos no se ven afectadas por las plantaciones establecidas en la reserva, debido a que estas llevan un proceso de regeneración avanzada, lo que las hace similares en la heterogeneidad, parámetro importante que determina la densidad de dichas comunidades al proporcionar gran variedad de recursos y hábitats.

- Las propiedades químicas M.O y $\mathrm{N}$ influyen en la diversidad de macroinvertebrados edáficos, ya que son variables que están correlacionadas positivamente con esta, lo que se ve reflejado en que la diversidad de Hill es similar en los sitios 
- La correlación positiva entre la densidad de macroinvertebrados edáficos y las variables estructurales de la plantación de eucalipto como área basal y diámetro a la altura del pecho, indica que la estructura vegetal de las plantas establecidas allí favorece la disponibilidad de recursos y de hábitats para la macrofauna, así como también refugio o lugar para completar su ciclo de vida.

\section{RECOMENDACIONES}

Para futuras investigaciones es conveniente comparar plantaciones forestales más jóvenes con un bosque natural de su misma edad en vez de un bosque secundario que se encuentre en proceso de regeneración muy avanzada, para determinar con más certeza su influencia sobre la macrofauna edáfica y sobre la calidad del ecosistema. Se recomienda estimar la calidad y tasas de descomposición de la hojarasca en el suelo de los sitios de estudio para complementar los resultados del contenido de materia orgánica, ya que pueden influir en la densidad de la macrofauna edáfica.

\section{AGRADECIMIENTOS}

Agradecemos a la Central Hidroeléctrica de Caldas (CHEC) por la financiación del proyecto. A Fabio Cupul, investigador titular del Departamento de Ciencias Biológicas del Centro Universitario de la Costa de la Universidad de Guadalajara en Puerto Vallarta, Jalisco, México; a la profesora Carmen Soledad Morales de la Universidad de Caldas por su valiosos aportes; y a los guardabosques de la reserva forestal protectora de la Central Hidroeléctrica de Caldas (CHEC).

\section{BIBLIOGRAFÍA}

ADIS, J., 2002.- Myriapoda: identification to classes: 457-554 (en) ADIS, J. (ed.), Amazonian Arachnida and Myriapoda. Identification keys to all classes, orders, families, some genera, and lists of known terrestrial species. Pensoft Publishers, Sofia.

ÁLVAREZ-MEJÍA, L.M., SANÍN, D., ALZATE-QUINTERO, N.F., CASTAÑO, N., MANCERA, J.C. \& GONZÁLEZ, G., 2007.- La flora de la región Centro-Sur de Caldas: 35-152 (en) Plantas de la región Centro-Sur de Caldas - Colombia. Editorial Universidad de Caldas, Manizales.

ANDERSON, J.M. \& INGRAM, J.S.I. (eds.), 1993.- Tropical soil biology and fertility: a handbook of methods. $\mathrm{CAB}$ International, Wallingford.

ANÓNIMO., 2003.- A Globe Learning Investigation. Land cover/Biology Investigation. The globe Program, Colorado, U.S.A.

ARNETT, R.H. \& THOMAS, M.C. (eds.), 2001.- American Beetles. Archostemata, Myxophaga, Adephaga, Polyphaga: Staphyliniformia. CRC Press LLC, Boca Ratón.

ARNETT, R.H., THOMAS, M.C., SKELLEY, P.E. \& FRANK, J.H. (eds.), 2002.- American Beetles. Polyphaga: Scarabaeoidea through Curculionoidea. CRC Press LLC, Boca Ratón.

AUGUST, P.V., 1983.- The role of habitat complexity and heterogeneity in structuring tropical mammal communities. Ecology, 64: 1495-1507.

BEGÓN, M., TOWNSEND, C.R. \& HARPER, J.L., 2006.- Patterns in Species Richness: 602-632 (en) BEGÓN, M., TOWNSEND, C.R. \& HARPER, J.L. (eds.) Ecology. From Individuals to Ecosystems. Blackwell Publishing Ltd.

BROCKERHOFF, E.G., JACTEL, H., PARROTTA, J.A., QUINE, C.P. \& SAYER, J., 2008.- Plantation forests and biodiversity: oxymoron or opportunity? Biodiversity Conservation, 17: 925-951.

CABRERA, G. \& CRESPO, G., 2001.- Influencia de la biota edáfica en la fertilidad de los suelos en ecosistemas de pastizales. Revista Cubana de Ciencia Agrícola, 35: 3-9.

CASTAÑO-VILLA, G.J., MORALES-BETANCOURT, J.A. \& BEDOYA-ÁLVAREZ, M.L., 2008.- Aportes de una plantación forestal mixta a la conservación de la avifauna en el cañón del río Cauca, Colombia. Revista Facultad Nacional de Agronomía, Medellín, 61: 4358-4365. 
CHAMORRO-BELlO, C., 2001.- El suelo: maravilloso teatro de la vida. Revista de la Academia Colombiana de Ciencias Exactas, Físicas y Naturales, 25: 483-494.

DECAËNS, T., JIMÉNEZ, J.J., GIOIA, C., MEASEY, G.J. \& LAVELLE, P., 2006.- The values of soil animals for conservation biology. European Journal of Soil Biology, 42: 23-38.

DE GOEDE, R.G.M., 1996.- Effects of sod-cutting on the nematode community of a secondary forest of Pinus sylvestris L. Biology and Fertility of Soils, 22: 227-236.

DOUBE, B.M. \& SCHMIDT, O., 1997.- Can the Abundance or Activity of Soil Macrofauna be used to Indicate the Biological Health of Soils?: 265-296 (en) PANKHURST, C.E., DOUBE, B.M. \& GUPTA, V.V.S.R. (eds.) Biological Indicators of Soil Health. Cab International, Wallingford.

ETIENNE, M. \& PRADO, C., 1982.- Descripción de la vegetación mediante la Cartografía de Ocupación de Tierras. Conceptos y manual de uso práctico. Editorial Universidad de Chile, Santiago.

FRAGOSO, C., REYES-CASTILLO, P. \& ROJAS, P., 2001.- La importancia de la biota edáfica en México. Acta Zoológica Mexicana, Número especial, 1: 1-10.

HARVEY, M.S., 1992.- The phylogeny and classification of the Pseudoscorpionida (Chelicerata: Arachnida). Invertebrate Taxonomy, 6: 1373-1435.

HERNÁNDEZ-ORTIZ, V. \& J. DZUL-CAUICH, J.F., 2008.- Moscas (Insecta: Diptera): 95-106 (en) MANSON, R.H., HERNÁNDEZ-ORTIZ, V., GALLINA, S. \& MEHLTRETER, K. (eds.) Agroecosistemas cafetaleros de Veracruz. Biodiversidad, manejo y conservación. Instituto de Ecología A.C. (INECOL) e Instituto Nacional de Ecología (INE-SEMARNAT), México.

HILL, M.O., 1973.- Diversity and evenness: a unifying notation and its consequences. Ecology, 54: 427-432.

HOLDRIDGE, L.R., 1967.- Life Zone Ecology. Tropical Science Center, San José.

HUERTA-LWANGA, E., RODRÍGUEZ-OLÁN, J., EVIA-CASTILLO, I., MONTEJO-MENESES, E., DE LA CRUZMONDRAGÓN, M. \& GARCÍA-HERNÁNDEZ, R., 2008.- Relación entre la fertilidad del suelo y su población de macroinvertebrados. Terra Latinoamericana, 26:171-181.

IRMLER, U., 2000.- Changes in the fauna and its contribution to mass loss and N release during leaf litter decomposition in two deciduous forests. Pedobiología, 44: 105-118.

JOCQUÉ, R. \& DIPPENAAR-SCHOEMAN, A.S., 2007.- Spider Families of the World. Royal Museum for Central Africa.

JOHNSTON, C.A., GROFFMAN, P., BRESHEARS, D.D., CARDON, Z.G., CURRIE, W., EMANUEL, W. et al., 2004.Carbon cycling in soil. Frontiers in Ecology and the Environment, 2: 522-528.

LAVELLE, P., 2000.- Ecological challenges for soil science. Soil Science, 165: 73-86.

LEÓN-GAMBOA, A.L., RAMOS, C. \& GARCÍA, M.R., 2010.- Efecto de plantaciones de pino en la artropofauna del suelo de un bosque altoandino. Revista de Biología Tropical, 58: 1031-1048.

LORANGER-MERCIRIS, G., IMBERT, D., BERNHARD-REVERSAT, F., PONGE, J.F. \& LAVELLE, P., 2007.- Soil fauna abundance and diversity in a secondary semi-evergreen forest in Guadeloupe (Lesser Antilles): influence of soil type and dominant tree species. Biology and Fertility of Soils, 44: 269-276.

MANLAY, R.J., MAGUETTE, K., MASSE, D., CHOTTE, J.L., CIORNEI, G. \& FLORET, C., 2002.- Carbon, nitrogen and phosphorus allocation in agro-ecosystems of a West African savanna. I. The plant component under semi-permanent cultivation. Agriculture, Ecosystems and Environment, 88: 215-232.

MENDOZA, A. \& OYAMA, K., 1999.- Ecology, management and conservation of potentially ornamental palms. Acta Horticulturae, 486: 79-86.

MORALES, J. \& SARMIENTO, L., 2002.- Dinámica de los macroinvertebrados edáficos y su relación con la vegetación en una sucesión secundaria en el páramo venezolano. Ecotrópicos, 15: 99-110.

MORENO, A.G., 2004.- Lombrices de tierra: material y métodos: 25-38 (en) MORENO, A.G. \& BORGES, S. (eds.) Avances en taxonomía de lombrices de tierra. Editorial Complutense y Obra Social Cajamadrid, Madrid.

MORENO, C.E., 2001.- Métodos para medir la biodiversidad. M\&T-Manuales y Tesis SEA, Zaragoza.

PARROTTA, J.A., TURNBULL, J.W. \& ONES, N., 1997.- Catalyzing native forest regeneration on degraded tropical lands. Forestry Ecology and Management, 99: 1-7.

STEHR, F.W., (ed.), 1987.- Immature Insects. Kendall/Hunt Publishing Company, Dubuque. (ed.), 1991.- Immature Insects. Kendall/Hunt Publishing Company, Dubuque.

STRESEMANN, E., 1986.- Exkursions- Fauna Von Deutschland.Wirbellose I. Volk und WissenVerlag, Berlin.

TRIPLEHORN, CH.A. \& JOHNSON, N.F., 2005.- Borror and Delong's Introduction to the study of Insects. Thomson Brooks/Cole.

WARREN, M.W. \& ZOU, X., 2002.- Soil macrofauna and litter nutrients in three tropical tree plantations on a disturbed site in Puerto Rico. Forest Ecology and Management, 170: 161-171.

WILLIAMS, A., KING, K., DANIEL, H. \& MARTIN, B., 2010.- Managing soil biological decline during longfallows in cropping systems. World Congress of Soil Science, Soil Solutions for a Changing World, 1-6 August, Brisbane, Australia.

ZALDÍVAR-SUÁREZ, N., BENÍTEZ-JIMÉNEZ, D., PÉREZ-MACHADO, B., FERNÁNDEZ-VERDECIA, Y., MONTECELOS-ZAMORA, Y. \& LICEA-CASTRO, L., 2009.- Efecto de la vegetación sobre la biodiversidad de macroinvertebrados del suelo en ecosistemas ganaderos. Revista Electrónica Granma Ciencia, 13: 1-8.

ZAPATA, R.D. \& OSORIO, N.W., 2010.- La materia orgánica del suelo: 357-396 (en) BURBANO-OJEDA, H. \& SILVA-MOJICA, F. (eds.) Ciencia del Suelo. Principios Básicos. Sociedad Colombiana de la Ciencia del Suelo, Bogotá. 